\title{
Successful Pregnancy and Delivery Following Two Heart Transplantations
}

\author{
Emily M Williams ${ }^{1}$, Karen L Florio ${ }^{1,3 *}$, Anna Grodzinsky2,4, Tara Banaszek Daming ${ }^{1}$ and Anthony Magalski ${ }^{2,4}$ \\ ${ }^{1}$ Department of Obstetrics and Gynecology, University of Missouri, USA \\ ${ }^{2}$ Department of Internal Medicine, University of Missouri, USA \\ ${ }^{3}$ Department of Maternal Fetal Medicine, Saint Luke's Hospital, USA \\ ${ }^{4}$ Saint Luke's Cardiovascular Consultants, Saint Luke's Hospital, USA \\ Submission: February 28, 2018; Published: April 20, 2018 \\ *Corresponding author: Karen L Florio, D0, Department of Maternal Fetal Medicine, Saint Luke’s Hospital of Kansas City, 4401 Wornall Rd, Peet \\ Center, Kansas City, MO, USA, 64111, Tel: 816-932-0270; Fax: 816-932-5873; Email: kflorio@saintlukeskc.org
}

\section{Abstract}

The number of post cardiac transplant patients desiring and achieving pregnancy continues to grow as more of these patients reach reproductive age. We present a case of a successful pregnancy and delivery in a 37 year old woman following a second heart transplantation. Given the complex clinical scenarios of both pregnancy and cardiac transplantation, we recommend a systematic and multidisciplinary approach for these patients.

\section{Introduction}

Janeway's lesions are non tender small erythematous or hemCardiac transplantation is a life saving procedure for those patients with end stage heart failure. Advances in the field of transplant over recent decades have improved survival outcomes for post cardiac transplant patients. Approximately one third of heart transplant recipients are women and of those, approximately $20 \%$ are of reproductive age $[1,2]$. Obstetric management of the post cardiac transplant patient is a complex clinical scenario with increasing incidence as these women continue to desire childbearing following surgery. Both pregnancy and the post transplant patient require specialized attention, and such pregnancies are considered high risk. Organ transplant increases pregnancy related morbidity including increased rates of spontaneous abortion, preterm birth, preeclampsia, and maternal mortality [3-5]. The physiologic changes of pregnancy may affect graft function. Beginning early in pregnancy, an increase in plasma volume as well as a change in the volume of distribution can affect circulating levels of anti rejection medications [3-5]. This can be compounded by nausea and vomiting, which affects up to $80 \%$ of patients in the first trimester [6]. The increase in cardiac output and stroke volume increase the workload of the graft. The hyperestrogenic state of pregnancy increases risks of thromboembolic events, which can lead to right heart strain [4]. Finally, the fetus itself has inherent immunogenic properties which may put the graft at risk for rejection.

\section{Case Report}

Our patient is a 37 year old G2P1001 who presented to our clinic for prenatal care at five weeks estimated gestational age. She reported a history of two cardiac transplantations; the first in 1997 for presumed viral dilated cardiomyopathy and the second in 2006 for end stage cardiac allograft vasculopathy. Her pregnancy was additionally complicated by tacrolimus -induced insulin dependent diabetes mellitus, hypertension, prior cesarean delivery, and herpes simplex virus.

Throughout her pregnancy she was followed by a multidisciplinary team including transplant physicians, cardiologists, endocrinologists, maternal-fetal medicine, anesthesiologists, and medical geneticists. A maternal echocardiogram (ECHO) was performed in the 1st trimester with normal ventricular size and function, no valvular abnormalities and an ejection fraction (EF) of $65 \%$. These findings were stable from her prior pregnancy. Maternal echocardiograms were repeated serially (every 2-4 months) throughout pregnancy to monitor for development of graft dysfunction and no significant changes developed. Throughout her gestation, the patient maintained NYHA class I functional status.

She was continued on her anti-rejection regimen of prednisone and tacrolimus. Her tacrolimus levels were monitored monthly to ensure that she remained in a therapeutic range. Blood glucose 
levels were reported weekly and insulin doses were titrated as indicated. Her diabetes was well controlled.

At nineteen weeks estimated gestational age, a detailed fetal anatomy ultrasound and fetal ECHO were performed and were both normal. She underwent serial fetal growth ultrasounds throughout pregnancy and antenatal testing was initiated at 32 weeks. Fetal growth was appropriate for gestational age.

At 36 weeks, she presented to labor and delivery with preterm premature rupture of membranes and was admitted for a repeat cesarean section which was performed without complications under regional anesthesia. She delivered a female infant with APGARS of 8 and 9, weighing 2515g, with no neonatal complications.

Her postpartum course was uncomplicated. Routine post transplant monitoring remained normal with normal ECHO, EKG and coronary angiography. Additionally, she decided to pursue genetic testing for hereditary cardiomyopathies postpartum (which she had previously declined), which revealed two mutations: lamin A/C (LMNA), a known pathogenic variant for dilated cardiomyopathy, as well as desmoplakin (DSP), a variant of unknown significance. Her offspring both tested positive for the LMNA variant.

\section{Discussion}

With the advances in transplant medicine, pregnancy is now a reality in women who were previously cautioned against childbearing. Although these pregnancies are not without their complications for both the mother and fetus, there are numerous reported cases in the literature of women carrying to term $[3,5]$. There are multiple case reports and case series describing outcomes for these patients, however there are no prospective or randomized trials to evaluate best practices.

The long-term effects of the hemodynamic stressors imposed by pregnancy in women with hereditary cardiomyopathies is not well understood. Unlike peripartum cardiomyopathy which is known to have up to a $20 \%$ relapse in subsequent pregnancies even with normal ventricular function, there are no such studies comparing outcomes in women with genetically-associated cardiomyopathy [7]. Although our patient maintained a normal function prior to and throughout gestation, it unknown whether her recent pregnancy will have a negative impact on long-term prognosis.

Here, we have reported on the first known successful pregnancy following a second heart transplantation. Additional investigation in the field of transplant medicine and pregnancy is warranted in order to further elucidate safety and efficacy of immunosuppressive agents, the long term effects of pregnancy on graft function, the effects of transplant on fertility, and optimization of contraception. Further, genetic testing may offer the subset of these patients with hereditary cardiomyopathies some control in family planning through interventions such as prenatal diagnosis or advanced reproductive technologies such as preimplantation genetics.

\section{Conclusion}

This is the first reported successful pregnancy in a patient who has undergone two separate heart transplants. While challenging, successful pregnancy outcomes following heart transplantation can be achieved by employing a multidisciplinary approach.

\section{References}

1. Kalinka J, Szubert M, Zdziennicki A, Chojnowski K, Maciejewski M, et al. (2014) A second delivery after heart transplantation - a case study. Kardiochir Torakochirurgia Pol 11(3): 339-342.

2. Qi X, Wang X, Wang C, Gu Y, Li Y, et al. (2016) Anesthesia management for cesarean section 10 years after heart transplantation: a case report. Springerplus 5(1): 993.

3. D'Souza RD, Acuna S, Zaffar N, Bhagra C, Ross H, et al. (2017) 758: Pregnancy outcomes of cardiothoracic transplant recipients: a systematic review and meta-analysis. American Journal of Obstetrics and Gynecology 216(1): S440.

4. Abdalla M, Mancini DM (2014) Management of pregnancy in the postcardiac transplant patient. Semin Perinatol 38(5): 318-325.

5. McKay DB, Josephson MA (2006) Pregnancy in recipients of solid organs --effects on mother and child. N Engl J Med 354(12): 12811293.

6. Matthews A, Haas DM, O’Mathúna DP, Dowswell T, Doyle M (2014) Interventions for nausea and vomiting in early pregnancy. Cochrane Database Syst Rev (3): CD007575.

7. Elkayam U (2014) Risk of subsequent pregnancy in women with a history of peripartum cardiomyopathy. J Am Coll Cardiol 64(15): 16291636.

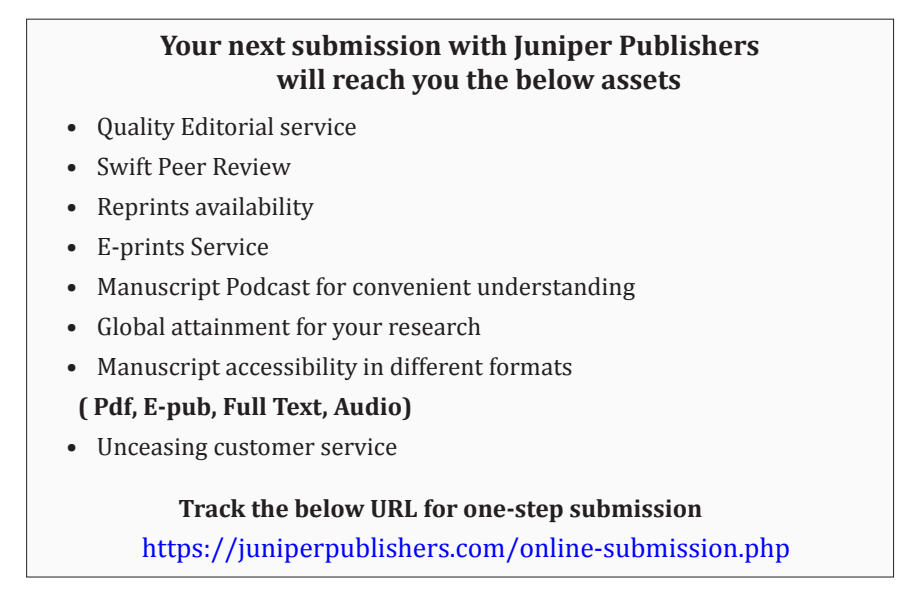

